\title{
ADAPTACIÓN A LA PRISIÓN Y AJUSTE PSICOLÓGICO EN UNA MUESTRA DE INTERNOS EN CENTROS PENITENCIARIOS
}

\author{
ADAPTATION TO PRISON AND \\ PSYCHOLOGICAL ADJUSTMENT IN A \\ SAMPLE OF PRISON INMATES
}

\author{
Mercedes Novo ${ }^{1}$, ANA Pereira ${ }^{2}$, MARÍA José VÁZQUEZ ${ }^{3}$ Y \\ BÁRBARA G. AMADO ${ }^{2}$
}

Cómo referenciar este artículo/How to reference this article:

Novo, M., Pereira, A., Vázquez, M. J. y Amado, B. G. (2017). Adaptación a la prisión y ajuste psicológico en una muestra de internos en centros penitenciarios [Adaptation to Prison and Psychological adjustment in a sample of prison inmates]. Acción Psicológica, 14(2), 113-128. https://doi.org/10.5944/ap.14.2.20784

\section{Resumen}

Aunque la literatura ha abordado ampliamente el estudio de la prisionización, el análisis de los efectos de las variables criminológicas en la adaptación a prisión y del estatus social en prisión, y de éstas en el ajuste psicológico y las destrezas cognitivas que emplean los internos no ha sido suficientemente trabajado. Para conocer de ello, evaluamos en variables criminológicas, adaptación a prisión, estatus social en prisión, ajuste psicológico y destrezas cognitivas a una muestra de 112 internos varo- nes en cumplimiento de una pena privativa de libertad de una prisión portuguesa, con edades entre 23 y 74 años $(M=40.40, D T=1.04), 77(68.8 \%)$ con antecedentes penales (reincidentes). Los resultados revelaron que las variables criminológicas (e.g., problemas en la familia) estaban relacionados con niveles más bajos de adaptación a la prisión. Además, los internos presentaban peor ajuste psicológico que la población general, y los reincidentes que los primarios. Asimismo, también pusieron de manifiesto que los internos se caracterizaban en la resolución de problemas por recurrir a estrategias de afrontamiento evitativas (i.e., Evitación cognitiva, Aceptación

Correspondencia: Mercedes Novo, Facultade de Psicoloxía. Universidade de Santiago de Compostela, España. Email: mercedes.novo@usc.es

ORCID: Mercedes Novo (http://orcid.org/0000-0002-0942-1501), Ana Pereira (http://orcid.org/0000-0002-3195-3504) y Bárbara G. Amado (http://orcid.org/0000-0001-9262-1469).

${ }^{1}$ Facultade de Psicoloxía, Universidade de Santiago de Compostela, España.

${ }^{2}$ Unidade de Psicoloxía Forense, Universidade de Santiago de Compostela, España.

${ }^{3}$ Departame nto AIPSE, Universidade de Vigo, España.

Recibido: 17 julio de 2017.

Aceptado: 19 de septiembre de 2017. 
o Resignación, Búsqueda de recompensas alternativas, Descarga emocional), en detrimento de las aproximativas (i.e., Análisis lógico). Se discuten las implicaciones de los resultados para el diseño de programas de intervención dentro de prisión y la preparación para la vida en libertad (i.e., tercer grado).

Palabras clave: Adaptación a prisión; Estatus social; Afrontamiento; Ajuste psicológico; Destrezas cognitivas.

\begin{abstract}
Although prisonization has been studied in previous literature, the effects of criminological variables on prison adaption and prison social status; and of the latter on psychological adjustment and cognitive skills have not been sufficiently analysed. As for knowing about this, 112 male prison inmates with an age range between 23 and 74 years $(M=40.40, S D=1.04), 77(68.8 \%)$ with criminal records (recidivists) serving sentences in a Portuguese prison were evaluated in prison adaption, prison social status, psychological adjustment and cognitive skills. The results showed that criminological variables (e.g., family problems) were related with lower levels of adaption to prison. Furthermore, prison inmates exhibited a significant worse psychological adjustment than the normative population, and recidivists than primary offenders. Likewise, the results revealed that prison inmates were characterized in problem solving tasks by adopting an avoidant coping style (i.e., Cognitive avoidance, Acceptance or Resignation, Seeking alternative rewards, Emotional discharge), at the expense of an approach coping style (i.e., Logical analysis). The implications of the results for in-prison intervention programmes design and the preparation for a third-degree life i.e., under parole, are discussed.
\end{abstract}

Keywords: Adaptation to prison; Social status; Coping; Psychological adjustment; Cognitive skills.

\section{Introducción}

En los últimos años, la investigación en el ámbito de las prisiones ha prestado atención al estudio de la adaptación de los internos. Ya en el año 1950, Clemmer introdujo el término prisionización, que define como el proceso de "aculturación" carcelaria que experimentan los internos. En esta dirección, Haney (2002) señala que la prisionización consiste en la incorporación de las normas de la prisión en los hábitos de pensar, sentir y actuar de los presos. Caldwell (1956) plantea que la prisionización se relaciona con el estatus social asignado al interno, ya que el ambiente carcelario demanda la aceptación de determinados patrones de conducta, acordes a la cultura de la prisión. Así, a cada recluso se le atribuye una posición en la escala social, o estatus social, que, o bien se le asigna (e.g., edad, raza, inteligencia, historial familiar); o bien, alcanza (e.g., condenas previas, ocupación dentro de la prisión, pertenencia a grupos informales de internos, tipo de visitas o la aceptación del código carcelario) (Caldwell, 1956). Más recientemente, y a pesar de la aparente ubicuidad del código carcelario, distintas investigaciones han puesto de manifiesto que los internos no lo siguen de manera uniforme. Sus normas o valores están, en cierto modo, sujetos a interpretación, siendo en muchos casos las contingencias sociales las que explican que la adherencia al código de la prisión sea variable (Copes, Brookman y Brown, 2016). En esta dirección, Crewe (2005) plantea que este sistema de creencias compartido se ha debilitado en importancia e intensidad, ya que se han producido cambios en la cultura carcelaria relacionados con la pérdida del sentido de comunidad, así como mejoras en las condiciones de las prisiones. Es más, los códigos de conducta carcelarios pueden ser recursos o explicaciones que los internos utilizan a posteriori para justificar su comportamiento, no tanto determinantes del mismo (Copes et al., 2016). Entre otras, se han planteado medidas de adaptación del interno a la prisión basándose en indicadores objetivos de carácter administrativo (Wolfgang, 1961), que han resultado eficaces en la evaluación del ajuste a la prisión (Brodsky y Smitherman, 2013).

Asimismo, el contexto carcelario tiene un efecto estructural en los internos y determina fuertemente su salud mental (Yang, Kadouri, Révah-Lévy, Mulvey y Falis- 
sard, 2009). En este sentido, Haney (2002) plantea que el proceso de prisionización puede incluir, a nivel psicológico, distintos cambios y adaptaciones, a saber: hipervigilancia, sospecha interpersonal y desconfianza, sobrecontrol emocional, alienación y distanciamiento psicológico, retraimiento social y aislamiento, incorporación de normas propias de la cultura carcelaria, disminución de autoestima y valor personal, estrés postraumático y dependencia de la estructura de la prisión. La investigación ha destacado el papel que desempeñan las estrategias de afrontamiento en el proceso de adaptación a la prisión (Brown y Ireland, 2006; Sappington, 1996; Zamble, 1992). Estas pueden definirse como los esfuerzos realizados para dominar, reducir o tolerar las exigencias creadas por el estrés que representa la pérdida de libertad (Weiten, Dunn y Hammer, 2011), y cuya demanda se incrementa cuando el interno ingresa en prisión (Edward, Zamble y Porporino, 1990; Picken, 2012). Así, la investigación sugiere que los esfuerzos de afrontamiento de los internos se caracterizan por ser no planificados, desordenados y esporádicos, siendo el déficit de afrontamiento un factor de riesgo para la reincidencia (Zamble y Porporino, 1988). Sin embargo, en cuanto a la relación entre estilos de afrontamiento y salud mental, los resultados son contradictorios (Kirchner, Forns y Mohíno, 2007). Algunos autores constatan que optar solo por estrategias de afrontamiento aproximativas no protege contra la psicopatología (Vitaliano, Russo y Maiuro, 1987), frente a otros estudios que informan de mayores niveles de psicopatología en los internos que emplean estrategias evitativas (Kirchner et al., 2007).

Teniendo en cuenta lo expuesto anteriormente, nos planteamos un estudio con el objetivo de conocer la adaptación a la prisión de los internos, y su relación con el ajuste psicológico y cognitivo.

\section{Método}

\section{Participantes}

Como participantes se reclutaron 112 internos en el establecimiento penitenciario Central de Paços de $\mathrm{Fe}$ rreira (Portugal) que se encontraban en cumplimiento de una pena privativa de libertad. Todos eran varones, con edades entre 23 y 74 años $(M=40.40, D T=1.04)$ y en su mayoría de raza caucásica ( $94 \%$ ). En cuanto al estado civil, el $28.6 \%$ (32) vivían en pareja (casados, unión de hecho, convivencia) y el $71.4 \%$ (80) solos (solteros, separados, viudos). En el momento de ingresar en el centro penitenciario, el 91\% había completado su formación en la escuela primaria, el $8 \%$ la secundaria, mientras el $2 \%$ tenía uno o más títulos universitarios. En el momento de la evaluación, el $71 \%$ había finalizado la escuela primaria, el $26 \%$ la secundaria y el $3 \%$ tenía uno o más títulos universitarios.

Setenta y siete $(68.8 \%)$ contaban con antecedentes penales (reincidentes), y la media del número de delitos por los que habían sido enjuiciados y condenados era de $8.57(D T=0.90)$, oscilando en rango entre 1 y 44 delitos. Los tipos penales, según el código portugués, se concretan en delitos contra la sociedad y la propiedad $(34.85 \%)$, contra las personas ( $16 \%)$, contra la sociedad $(11.6 \%)$, contra la sociedad y las personas $(9.8 \%)$, contra la propiedad ( $8 \%)$, contra la propiedad y las personas $(6.3 \%)$ y un $13.4 \%$ que cumplen condena por más de dos tipos delictivos.

El tiempo medio de permanencia en esta institución fue 4.40 años $(D T=2.25)$. En cuanto al tiempo de condena cumplido de la sentencia impuesta, el promedio se sitúa en 6.42 años $(D T=3.16)$, siendo la longitud media de la sentencia de pena privativa de libertad de 13.20 años $(D T=5.18)$. La edad media al primer arresto fue 29.29 años $(D T=0.06)$. De la muestra evaluada, el $61.60 \%$ tenían delitos anteriores, y por tanto eran reincidentes, frente al $38.46 \%$ que no lo eran.

\section{Procedimiento y diseño}

Los sujetos participaron de forma voluntaria. El diseño del estudio contó con la aprobación previa y el permiso de los centros para la evaluación, así como del consentimiento por escrito de los internos evaluados. Sometida a análisis la sensibilidad del diseño, la probabilidad de detección $(1-\beta)$ de diferencias significativas $(\alpha<.05)$ y para un tamaño del efecto medio, oscilaba, según el análisis de datos, entre el $84 \%$ y el $99 \%$. En conse- 
cuencia, el diseño ejecutado es altamente sensible en la detección de diferencias significativas.

\section{Instrumentos de medida}

Los expedientes penitenciarios de los internos aportaron la información sociodemográfica y procesal-penal de la situación del interno (variables criminológicas). Por su parte, como instrumento de medida de la psicopatología se tomó el SCL-90-R (Derogatis, 1994), que evalúa nueve dimensiones clínicas (i.e., somatización, obsesivocompulsivo, sensibilidad interpersonal, depresión, ansiedad, hostilidad, ansiedad fóbica, ideación paranoide, psicoticismo) y tres indicadores globales de severidad (i.e., Índice de Severidad Global [GSI], Total de Síntomas Positivos [PST], Índice de Malestar Referido a Síntomas Positivos [PSDI]). Este instrumento fue sometido a un proceso de traducción inversa del español al portugués.

Con los participantes en el estudio, el análisis de la consistencia interna del SCL-90 reportó una alta consistencia interna para las escalas clínicas (con los participantes en el estudio, $\alpha=.801$ para la escala de somatización; $\alpha=.680$ para obsesivo-compulsivo; $\alpha=.731$ para sensibilidad interpersonal; $\alpha=.716$ para depresión; $\alpha=.771$ para ansiedad; $\alpha=.674$ para hostilidad; $\alpha=.679$ para ansiedad fóbica; $\alpha=.724$ para ideación paranoide, $y$ $\alpha=.677$ para psicoticismo).

Con el objetivo de evaluar la capacidad de afrontamiento recurrimos al CRI-A (Coping Responses Inventory Adult Form) de Moos (1993), que se compone de dos partes. En la primera se solicita al evaluado que piense sobre el problema más importante por el que haya pasado en los últimos 12 meses o, en su defecto, en un problema menor que haya tenido que enfrentar y, a continuación, ha de responder a 10 preguntas sobre el problema para las cuales existen cuatro alternativas de respuesta: nunca, casi nunca, a menudo y siempre. En la segunda parte, se formulan otras 48 preguntas relativas al mismo problema, con cuatro alternativas de respuesta: nunca, pocas veces, algunas veces y bastantes veces. En ambas formas, el interno fue instruido para contestar a la prueba tomando como situación-problema el ingreso en prisión. El CRI-A evalúa ocho tipos de estrategias de afrontamiento, que se mostraron fiables con los participantes en el estudio: Análisis lógico (LA) $(\alpha=.771)$, que se refiere a intentos cognitivos de entender y prepararse mentalmente para un estresor y sus consecuencias; Reencuadre positivo (PR) $(\alpha=.638)$ que mide intentos cognitivos de construir y reestructurar un problema de una manera positiva al mismo tiempo que se acepta la realidad de la situación; Búsqueda de orientación y apoyo (SG) $(\alpha=.676)$ que evalúa intentos comportamentales de buscar información, asesoramiento y apoyo; Resolución de problemas (PS) $(\alpha=.710)$ que valora intentos comportamentales para tomar parte en la resolución directa del problema; Evitación cognitiva (CA) $(\alpha=.790)$ que se relaciona con intentos cognitivos para evitar pensar de manera realista en el problema; Aceptación resignada (AR) $(\alpha=.702)$ que mide intentos cognitivos de reaccionar ante el problema aceptándolo; Búsqueda de actividades alternativas (SR) $(\alpha=.793)$ que aprecia intentos comportamentales de involucrarse en actividades sustitutivas y crear nuevas formas de satisfacción; Descarga emocional (ED) $(\alpha=.810)$ que evalúa intentos comportamentales de reducir la tensión expresando sentimientos negativos.

Para examinar el estatus social, se aplicó la Escala MacArthur de Estatus Subjetivo Social (Adler y Stewart, 2007), en la que se pide a los internos que se asignen en una escala de diez puntos, el lugar que ocupan dentro de la prisión en relación a otros internos del centro penitenciario, conformando el estatus social percibido. Por otro lado, se evaluó el estatus social externo de los reclusos, solicitando a los técnicos de la prisión que puntuasen a cada interno en la escala de de diez puntos, mediante una versión adaptada basada en los criterios de estatus social definidos por Caldwell (1956).

Finalmente, para valorar la adaptación a la cárcel, se utilizó el Prison Adjustment Index (PAI) de Wolfgang (1961), que evalúa el grado de adaptación del interno desde una perspectiva administrativa, basada en criterios tales como el número de ocupaciones dentro de la prisión y el tiempo en cada una de ellas, el número de destituciones del puesto y el número de infracciones registradas en la prisión. Así, del cómputo total de criterios el in- 
terno puede obtener una puntuación total que, si es positiva o negativa, es indicativa de una buena o mala adaptación. Concretamente, la información necesaria para evaluar estos criterios se recabó de los expedientes de los internos y fue contrastada con los técnicos de la prisión.

\section{Resultados}

\section{Adaptación a la prisión y variables criminológicas}

Aquellos internos que cuentan en su historial con problemas se informa de problemas comportamentales precoces $(M=0.43)$ informan de menor adaptación a la prisión, $t(110)=2.18, p<.05, d=0.48$, que aquellos en los que no se han registrado este tipo de problemas $(M=0.66)$. Además, los internos con historial de abuso $(M=0.45)$ también exhiben menor adaptación a la prisión, $t(107.05)=3.07, p<.01, d=0.62$, que aquellos que no tienen historial de abuso $(\mathrm{M}=0.73)$.

Asimismo, se hallaron diferencias en la adaptación de los internos a la prisión mediadas por el historial familiar de problemas psicoemocionales, $t(90.19)=1.92, p<.05$, $d=0.40$. Los internos con peor adaptación al contexto de la cárcel cuentan con un historial de este tipo de problemas en la familia $(M=0.48)$, en relación a los que no $(M=0.67)$. Además, se constató que los que vivían solos $(M=0.50)$ se adaptaban peor a la prisión, que aquellos que tenían pareja $(M=0.84), \mathrm{t}(77.47)=3.99, p<.001$, $d=0.77)$.

Así, un historial de comportamiento de riesgo, la existencia de problemas comportamentales precoces y de problemas psicoemocionales en el contexto familiar, se asocian con un peor ajuste al ambiente carcelario, mientras que el disponer de una relación de pareja aparece como un factor protector frente a la inadaptación a la prisión.

Por otra parte, los resultados revelan una relación significativa entre la adaptación a la prisión y el estatus social externo, $\chi^{2}(1, N=112)=3.57, p<.05$, phi $=.18$.
Así, el $68 \%$ de los internos bien adaptados tienen un estatus externo elevado, esto es, otorgado por los técnicos frente al $32 \%$ de los no adaptados. Para los que obtienen un bajo estatus, el $51 \%$ se clasifican en adaptados, frente al $49 \%$ de no adaptados. Sin embargo, la discrepancia entre la evaluación externa realizada por los técnicos y el estatus social autoinformado por los internos $t(112)=4.39, p<.001, d=0.42$, advierte que los penados comparten una autopercepción positivamente sesgada de su estatus social dentro de la prisión $(M=1.01$, IC95\%[0.56, 1.46]), aunque la variabilidad es muy alta $(D T=2.43, \quad C V=240.59)$. Este resultado refleja que el estatus social autopercibido se basa en el cumplimiento del código y valores carcelarios, mientras el externo en la adaptación y ajuste a la prisión.

\section{Ajuste psicológico}

El contraste de la psicopatología observada entre la población de internos en prisión con la población normativa (muestra de contraste al tratarse de delincuentes y no enfermos mentales) puso de manifiesto que los internos presentan sintomatología significativa (véase la Tabla 1) propia de somatización (e.g., disfunciones cardiovasculares, gastrointestinales, respiratorias), obsesióncompulsión (i.e., pensamientos, impulsos y acciones que son experimentados como imposibles de evitar o no deseados), sensibilidad interpersonal (v. gr., sentimientos de inferioridad e inadecuación, hipersensibilidad a las opiniones y actitudes ajenas, incomodidad e inhibición en las relaciones interpersonales), depresión (ad exemplum, estado de ánimo disfórico, falta de motivación, poca energía, sentimientos de desesperanza, ideas autodestructivas), ansiedad (verbigracia, nerviosismo, tensión, ataques de pánico y miedos), hostilidad (por ejemplo, pensamientos, sentimientos y conductas propios de estados de agresividad, ira, irritabilidad, rabia y resentimiento), ansiedad fóbica (esto es, miedo irracional y desproporcionado a personas concretas, lugares, objetos y situaciones), ideación paranoide (i. e., suspicacia, centralismo auto-referencial e ideación delirante, hostilidad, grandiosidad, miedo a la pérdida de autonomía y necesidad de control), y psicoticismo (o sea, alienación social al no ser población psiquiátrica). Del mismo modo, la población de 
Tabla 1

Escalas Básicas de Evaluación clínica del SCL-90. Valores " $t$ ” para una muestra con valor de contraste la media de grupo normativo

\begin{tabular}{|c|c|c|c|c|c|}
\hline Variable & $M_{\mathrm{PP}}$ & $M_{\mathrm{PG}}$ & $t$ & $p$ & $d$ \\
\hline \multicolumn{6}{|l|}{ Dimensiones clínicas } \\
\hline Somatización & 0.74 & 0.36 & 6.84 & .000 & 0.74 \\
\hline Obsesivo-Compulsivo & 1.24 & 0.39 & 16.11 & .000 & 1.67 \\
\hline Susceptibilidad Interpersonal & 0.88 & 0.29 & 10.43 & .000 & 1.18 \\
\hline Depresión & 1.01 & 0.36 & 13.43 & .000 & 1.35 \\
\hline Ansiedad & 0.79 & 0.30 & 9.18 & .000 & 1.03 \\
\hline Hostilidad & 0.58 & 0.30 & 5.24 & .000 & 0.57 \\
\hline Ansiedad Fóbica & 0.30 & 0.13 & 4.11 & .000 & 0.45 \\
\hline Ideación Paranoide & 1.72 & 0.34 & 22.21 & .000 & 2.46 \\
\hline Psicoticismo & 0.52 & 0.14 & 8.53 & .000 & 0.99 \\
\hline \multicolumn{6}{|l|}{ Índices Globales de Malestar } \\
\hline Índice Severidad Global (GSI) & 0.89 & 0.31 & 14.52 & .000 & 1.57 \\
\hline Total Síntomas Positivos (PST) & 35.38 & 19.29 & 10.68 & .000 & 1.02 \\
\hline Índice Malestar Síntomas Positivos (PSDI) & 2.22 & 1.32 & 24.91 & .000 & 2.25 \\
\hline
\end{tabular}

Nota. $g /(111)$; MPP: Media de la población penitenciaria; $M_{\mathrm{PG}}$ : Valor de prueba o contraste tomado de la Media de la población general del manual del SCL-90-R.

internos (ver Tabla 1) evidenció padecer un mayor malestar clínico global (GSI), más síntomas positivos (PST) y una mayor severidad en los síntomas positivos (PSDI) que la general. En suma, la población penitenciaria presenta más daños en las dimensiones clínicas que también se manifiesta en los índices de malestar.

Por su parte, el factor reincidencia (reincidentes vs. primarios) conlleva a diferencias de medias en la dimen- sión clínica obsesivo-compulsivo, que es significativamente mayor entre los penados reincidentes (ver Tabla 2 ); esto es, sufren de más pensamientos, impulsos y acciones que son experimentados como imposibles de evitar o no deseados. En las restantes dimensiones y en los índices de malestar, primarios y reincidentes comparten el mismo estado de salud mental.

Tabla 2

Escalas Básicas de Evaluación clínica del SCL-90. Valores "t" para muestras independientes. Variable de agrupación reincidencia (primarios vs. reincidentes)

\begin{tabular}{lrrrrr}
\hline Variable & $\boldsymbol{M}_{\mathbf{P}}$ & \multicolumn{1}{c}{$\boldsymbol{M}_{\mathbf{R}}$} & $\boldsymbol{t}(\boldsymbol{g l})$ & $\boldsymbol{p}$ & $\boldsymbol{d}$ \\
\hline Dimensiones clínicas & & & & & \\
Somatización & 0.68 & 0.77 & $-0.81(109.66)$ & .417 & 0.03 \\
Obsesivo-Compulsivo & 1.10 & 1.33 & $-2.17(110)$ & .033 & 0.06 \\
Susceptibilidad Interpersonal & 0.87 & 0.87 & $0.04(110)$ & .970 & 0.11 \\
Depresión & 1.02 & 1.01 & $0.15(110)$ & .874 & 0.00 \\
Ansiedad & 0.71 & 0.82 & $-0.99(110)$ & .325 & -0.08 \\
Hostilidad & 0.52 & 0.61 & $0.87(110)$ & .385 & -0.03 \\
Ansiedad Fóbica & 0.29 & 0.30 & $-0.11(110)$ & .915 & 0.19 \\
Ideación Paranoide & 1.71 & 1.73 & $-0.097(110)$ & .923 & 0.00 \\
Psicoticismo & 0.53 & 0.52 & $0.04(110)$ & .965 & 0.03 \\
Índices Globales de Malestar & & & & \\
İndice Severidad Global (GSI) & 0.84 & 0.90 & $-0.76(110)$ & .448 & 0.02 \\
Total Síntomas Positivos (PST) & 34.65 & 35.82 & $-0.38(110)$ & .706 & 0.04 \\
Índice Malestar Síntomas Positivos (PSDI) & 2.21 & 2.23 & $-0.30(110)$ & .762 & 0.39 \\
\hline
\end{tabular}

Nota. MP: Media del grupo internos primarios; $M_{R}$ : Media del grupo de internos reincidentes. 
Tabla 3

Escalas Básicas de Evaluación clínica del SCL-90. Valores "t” para muestras independientes. Variable de agrupación adaptación a la prisión

\begin{tabular}{|c|c|c|c|c|c|}
\hline Variable & $M_{\mathrm{a}}$ & $M_{\mathrm{n}-\mathrm{a}}$ & $t$ & $p$ & $d$ \\
\hline \multicolumn{6}{|l|}{ Dimensiones clínicas } \\
\hline Somatización & 0.71 & 0.76 & -0.42 & .672 & -0.08 \\
\hline Obsesivo-Compulsivo & 1.25 & 1.24 & 0.09 & .928 & 0.01 \\
\hline Susceptibilidad Interpersonal & 0.93 & 0.83 & 0.81 & .419 & 0.01 \\
\hline Depresión & 1.04 & 1.00 & 0.32 & .748 & 0.08 \\
\hline Ansiedad & 0.80 & 0.77 & 0.30 & .760 & 0.05 \\
\hline Hostilidad & 0.65 & 0.53 & 1.13 & .250 & 0.03 \\
\hline Ansiedad Fóbica & 0.32 & 0.28 & 0.42 & .670 & 0.09 \\
\hline Ideación Paranoide & 1.72 & 1.73 & -0.09 & .925 & 0.00 \\
\hline Psicoticismo & 0.52 & 0.53 & -0.17 & .859 & -0.02 \\
\hline \multicolumn{6}{|l|}{ Índices Globales de Malestar } \\
\hline Índice Severidad Global (GSI) & 0.90 & 0.87 & 0.41 & .684 & 0.07 \\
\hline Total Síntomas Positivos (PST) & 35.44 & 35.32 & 0.04 & .970 & 1.78 \\
\hline Índice Malestar Síntomas Positivos (PSDI) & 2.27 & 2.20 & 0.78 & .440 & 0.18 \\
\hline
\end{tabular}

Nota. $g /(112) ; M_{\mathrm{a}}$ : Media del grupo de adaptados; $M_{\mathrm{n}-\mathrm{a}}$ : Media del grupo de no-adaptados.

Tal y como se refleja en la Tabla 3, el factor adaptación a la prisión (adaptados vs. no-adaptados) no media diferencias en el ajuste psicológico, ni en los indicadores de malestar.

\section{Destrezas cognitivas para la resolución de problemas}

El afrontamiento incluye cuatro estrategias de aproximación al problema (i.e., Análisis lógico, Reevaluación positiva, Búsqueda de guía y soporte, Solución de pro- blemas) y cuatro de evitación del mismo (i.e., Evitación cognitiva, Aceptación o Resignación, Búsqueda de recompensas Alternativas, Descarga emocional). Los resultados del contraste de la capacidad para la resolución de problemas de los internos en contraste con la población general (ver Tabla 4) advierten que los internos recurren significativamente más a las estrategias evitativas de resolución de problemas (i.e., Evitación cognitiva, Aceptación o Resignación, Búsqueda de recompensas alternativas, Descarga emocional) que la población general, y significativamente menos a la aproximativa 'Análisis lógico'.

Tabla 4

Comparación de medias en estrategias de afrontamiento

\begin{tabular}{lcccc}
\hline Variable & $\boldsymbol{M}_{\mathrm{PP}}$ & $\boldsymbol{t}$ & $\boldsymbol{p}$ & $\boldsymbol{d}$ \\
\hline Análisis lógico & 46.25 & -3.46 & .001 & -0.65 \\
Reencuadre positivo & 51.08 & 1.30 & .196 & 0.24 \\
Búsqueda de orientación y apoyo & 48.95 & -1.03 & .302 & -0.19 \\
Resolución de problemas & 49.20 & -0.93 & .352 & -0.18 \\
Evitación cognitiva & 57.49 & 9.83 & .000 & 1.86 \\
Aceptación o resignación & 58.85 & 10.86 & .000 & 2.05 \\
Búsqueda de actividades alternativas & 60.85 & 10.77 & .000 & 11.50 \\
Descarga emocional & 61.42 & 13.55 & .000 & 11.61 \\
\hline
\end{tabular}

Nota. $g /(1,111) ;$ MP: Media de la población penitenciaria; VP: Valor de prueba o contraste tomado de la media de la población general del manual del CRI-A ( $\mathrm{T}=50)$. 


\section{Discusión}

Con anterioridad a la discusión cabe señalar algunas limitaciones propias de este estudio. Así, contamos con una muestra homogénea, en la que no se ha controlado el efecto de maduración de los internos en régimen ordinario (dentro de prisión) a lo largo de la condena, o el impacto que pueda tener el tratamiento penitenciario, en aquellos internos que se benefician de él mediante programas de intervención dentro de la prisión (Rocheleau, 2013). Además, este tipo de población es tendente a la manipulación de las respuestas, tanto en dirección a la simulación (American Psychiatric Association, 2014) para evitar sanciones u obtener una mejor clasificación penitenciaria, como a la disimulación, bien dirigida a la ocultación de características negativas (Arce, Fariña, Seijo y Novo, 2015), bien a la asunción de características positivas (Fariña, Redondo, Seijo, Novo y Arce, 2017). Esto último para obtener beneficios penitenciarios tal como permisos, destinos en prisión o el tercer grado. Sobre la base de estas limitaciones que median la generalización de los resultados, de los hallazgos previos se desprenden las siguientes conclusiones.

En lo que atañe al estatus y sus diferentes perspectivas de evaluación, los internos que, según la perspectiva de los técnicos, obtienen un estatus social más elevado, refieren una buena adaptación. Sin embargo, se verifica una discrepancia entre estatus social autoinformado y la evaluación externa, advirtiéndose que los penados comparten una percepción de su estatus social sesgada positivamente. Esta discrepancia puede tener relación con la atribución del estatus social dentro de la prisión. Así, siguiendo el código de la cárcel, el estatus social está determinado por distintos factores, entre ellos, la existencia de antecedentes penales (reincidencia) o el propio cumplimiento del código penitenciario (Caldwell, 1956), código este que salvaguarda la lealtad entre internos, desalentado la denuncia y la comunicación con la autoridad (Liebling y Crewe, 2012). La autoridad representada por los funcionarios, parece tener una valencia positiva en la atribución de un estatus elevado externo y asignado por los técnicos, y una valencia negativa, en la autopercepción. En este sentido, cabe mencionar que la aceptación del código carcelario se puede ver reducido por los cambios en la cultura carcelaria relacionados con la pérdida del sentido de comunidad, así como mejoras en las condiciones de las prisiones (Crewe, 2005). Así, el tratamiento penitenciario puede incidir mediante programas de intervención que mejoren el clima social y la convivencia, pero de manera particular que contribuyan a la reducción del impacto del código carcelario, siendo este aspecto crucial para el proceso de rehabilitación (Dettbarn, 2012).

En lo que se refiere a la adaptación de los internos, se observa que el contexto carcelario conlleva más desajuste para los sujetos de mayor vulnerabilidad al ingreso en prisión, esto es, aquellos que presentan, por ejemplo, abuso de sustancias, problemas psicoemocionales en el contexto familiar o comportamentales precoces, mientras que el disponer de una relación marital facilita dicha adaptación (Jiang y Winfree, 2006). En este sentido, señalar que este resultado respalda el modelo de importación, el cual plantea que la cultura carcelaria importa hábitos, valores y costumbres que la persona que entra en prisión ha adquirido previamente, y que influyen en los niveles de prisionización que presenta (Dhami, Ayton y Loewenstein, 2007; Paterline y Petersen, 1999). A este respecto, es necesario incidir en un tratamiento basado en una adecuada evaluación de necesidades y carencias de los internos en la fase de inicio de la intervención (Arce y Fariña, 2010), para diseñar una intervención multimodal y multinivel que responda a su vez a un modelo estructurado y explícito (Andres y Bonta, 2010), y en el que se atienda a las necesidades tanto criminógenas, que se relacionan con las causas de la violencia (Fariña, Arce y Novo, 2008), como no criminógenas, que se vinculan con la reincidencia (Arce, Fariña y Novo, 2014).

En términos de ajuste psicológico, los resultados indican que la población penitenciaria se diferencia de la población general en todas las variables clínicas evaluadas, así como en los tres índices de severidad, esto es, presenta más sintomatología clínica y ésta de mayor intensidad, aunque no se relaciona con el grado de adaptación a la prisión. Este resultado corrobora estudios previos que dan cuenta de una mayor prevalencia de alteraciones psicopatológicas en la población de internos (Arce, Fariña y Vázquez, 2011; Fazel y Danesh, 2002; Zabala-Baños et al., 2016) o mayor riesgo de depresión (Fazel 
y Seewald, 2012; Yang et al., 2009). Estos cambios y adaptaciones parecen responder al proceso de prisionización (Haney, 2002) (e.g., hipervigilancia, desconfianza personal y sospecha, sobrecontrol emocional, alienación y distanciamiento psicológico, retraimiento social, disminución de la autoestima, etc). En este sentido, algunos autores han informado que la salud mental de los internos si bien empeora al ingreso en prisión, al tiempo que transcurre la condena experimenta una mejoría (Walker et al., 2014), de manera que, por ejemplo, la depresión y la ideación suicida disminuyen transcurrido el primer año (Smyth, Ivanoff y Jang, 1994). Sin embargo, si bien el ajuste puede mejorar con la adaptación a la prisión, especialmente en las cárceles de menor tamaño, resta por analizar si en estos casos esa mejoría se mantiene al salir de la prisión (Walker et al., 2014). Además, las prisiones deberían responsabilizarse de una adecuada atención a la salud mental de los internos (Moller, Stover, Jurgens, Gatherer y Nikogosian, 2007), pudiendo considerarse el ingreso en prisión (Pérez, Herrero, Velasco y Rodríguez-Díaz, 2015), como una oportunidad para la intervención y mejora de la salud (Powell, Harris, Condon y Kemple, 2010), en aplicación de los principios de la Justicia Terapéutica acerca de los victimarios en prisión (Asociación Iberoamericana de Justicia Terapéutica, 2017). Es más, la salud mental puede considerarse un prerrequisito para una adecuada integración y preparación para la vida en libertad (Jordan, 2011). En todo caso, es necesaria más investigación que aborde la relación entre salud mental y prisión como un objetivo central (Walker et al., 2014).

Los resultados obtenidos revelan que los internos usan mayor medida estrategias evitativas, esto es, evitación cognitiva, aceptación o resignación, búsqueda de recompensas alternativas y descarga emocional. Este estilo de afrontamiento evitativo y centrado en las emociones, se caracteriza por la tendencia a hacerse ilusiones, evitar pensar y sentir estas situaciones como reales, al tiempo que favorece emociones negativas como la ira (PastwaWojciechowska, Kaźmierczak y Błażek, 2012). Por otra parte, en cuanto a las aproximativas, en relación a la población general hacen menos uso del análisis lógico. En suma, los penados utilizan estrategias desadaptativas (evitativas) en la resolución de problemas, en detrimento de las adaptativas (aproximativas) (Feelgood, Cortoni y Thompson, 2005).

En definitiva, este trabajo realiza algunas contribuciones al estudio de la adaptación y ajuste de los internos a la prisión, que pueden resultar de interés para el diseño de los programas de intervención en régimen ordinario (dentro de prisión) y de preparación para la vida en libertad, que han demostrado su eficacia en la mejora de la competencia social y cognitiva, tanto en adultos como en menores (Arce et al., 2014; Arce, Seijo, Fariña y Mohamed-Mohand, 2010), así como en la disminución de los efectos de la prisionización (Dettbarn, 2012).

\section{Referencias}

Adler, N. E. y Stewart, J. (2007). The MacArthur Scale of Subjective Social Status. MacArthur Research Network on SES and Health at UCSF. Recuperado de

http://www.macses.ucsf.edu/research/psychosocial/ subjective.php

American Psychiatric Association. (2014). Manual diagnóstico y estadístico de los trastornos mentales $(D S M-V)$ [Diagnostic and Statistical Manual of Mental Disorders (DSM-V)]. Madrid, España: Panamericana.

Andrews, D. y Bonta, J. (2010). The Psychology of Criminal Conduct (5a Ed.). New Providence, NJ: Matthew Bender and Company.

Arce, R. y Fariña, F. (2010). Diseño e implementación del Programa Galicia de Reeducación de Maltratadores: Una respuesta psicosocial a una necesidad social y penitenciaria [Design and Implementation of the Galician Program for Batterers' Re-Education: A Psychosocial Answer to a Social and Penitentiary Need]. Psychosocial Intervention, $19, \quad 153-166$. https://oi.org/10.5093/in2010v19n2a7 
Arce, R., Fariña, F. y Novo, M. (2014). Competencia cognitiva en penados primarios y reincidentes: Implicaciones para la reeducación [Cognitive Competence among Recidivist and Non-Recidivist Prisoners: Implications for the Rehabilitation]. Anales de Psicología, 30, 259-266. https://doi.org/10.6018/analesps.30.1.158201

Arce, R., Fariña, F. y Vázquez, M. J. (2011). Grado de competencia social y comportamientos antisociales delictivos y no delictivos en menores [Social competence and Delinquent, Antisocial, and NonDeviant Behavior in Adolescents]. Revista Latinoamericana de Psicología, 43, 473-486. https://doi.org/10.14349/rlp.v43i3.487

Arce, R., Fariña, F., Seijo, D. y Novo, M. (2015). Assessing Impression Management with the MMPI2 in Child Custody Litigation. Assessment, 22, 769777. https://doi.org/10.1177/1073191114558111

Arce, R., Seijo, D., Fariña, F. y Mohamed-Mohand, L. (2010). Comportamiento antisocial en menores: Riesgo social y trayectoria natural de desarrollo [Antisocial Behavior in Adolescents: Social Risk and Natural Developmental Trajectory]. Revista Mexicana de Psicología, 27(2), 127-142.

Asociación Iberoamericana de Justicia Terapéutica. (2017). Libro de conclusiones del III Congreso Iberoamericano de Justicia Terapéutica [Book of Conclusions of the III Ibero-American Therapeutic Justice Congress]. Santiago de Compostela, España: Andavira.

Brodsky, S. y Smitherman, H. N. (1983). Search and Selection of Scales for Review. En S. Brodsky y Smitherman, H. N. (Eds.), Handbook of Scales for Research in Crime and Delinquency (pp.1-8). New York, NY: Plenum Press.

Brown, S. L. e Ireland, C. A. (2006). Coping Style and Distress in Newly Incarcerated Male Adolescents. Journal of Adolescent Health, 38, 656-661. https://doi.org/10.1016/j.jadohealth.2005.09.005
Caldwell, M. G. (1956). Group Dynamics in the Prison Community. The Journal of Criminal Law, Criminology, and Police Science, 46, 648-657. https://doi.org/10.2307/1139377

Clemmer, D. (1958). The Prison Community (Rev. Ed.). New York, NY: Holt, Rinehart and Wintson.

Copes, H., Brookman, F. y Brown, A. (2016). Accounting for Violations of the Convict Code. Deviant Behavior, 34, 841-858. https://doi.org/10.1080/01639625.2013.781444

Crewe, B. (2005). Codes and Conventions: The Terms and Conditions of Contemporary Inmate Values. En A. Liebling, A. y S. Maruna. (Eds.), The Effects of Imprisonment (pp. 177-208). Portland, OR: Willan Publishing.

Dhami, M. K., Ayton, P. y Loewenstein, G. (2007). Adaptation to Imprisonment: Indigenous or Imported? Criminal Justice and Behavior, 34, 10851100. https://doi.org/10.1177/0093854807302002

Derogatis, L. R. (1994). SCL-90-R: Administration, Scoring and Procedures Manual ( $3^{\text {th }}$ Ed.). Minneapolis, MN: NCS Pearson.

Dettbarn, E. (2012). Effects of Long-Term Incarceration: A Statistical Comparison of two Expert Assessments of two Experts at the Beginning and the End of Incarceration. International Journal of Law and Psychiatry, 35, 236-239. https://doi.org/10.1016/j.ijlp.2012.02.014

Fariña, F., Arce, R. y Novo, M. (2008). Neighborhood and Community Factors: Effects on Deviant Behavior and Social Competence. The Spanish Journal of Psychology, 11, 78-84. https://doi.org/10.1017/S1138741600004133

Fariña, F., Redondo, L., Seijo, D., Novo, M. y Arce, R. (2017). A Meta-Analytic Review of the MMPI Validity Scales and Indexes to Detect Defensiveness in Custody Evaluations. International Journal of Clinical and Health 
Psychology

17 ,

https://doi.org/10.1016/j.ijchp.2017.02.002

Fazel, S. y Danesh, J. (2002). Serious Mental Disorder in 23000 Prisoners: A Systematic Review of 62 Surveys. The Lancet, 359, 545-550. https://doi.org/10.1016/S0140-6736(02)07740-1

Fazel, S. y Seewald, K. (2012). Severe Mental Illness in 33588 Prisoners Worldwide: Systematic Review and Meta-Regression Analysis. The British Journal of Psychiatry, 20, 364-373. https://doi.org/10.1192/bjp.bp.111.096370

Feelgood, S., Cortoni, F. y Thompson, A. (2005). Sexual Coping, General Coping, and Cognitive Distortions in Incarcerated Rapist and Child Molesters. Journal of Sexual Aggression, 11, 157-170. https://doi.org/10.1080/13552600500073657

Haney, C. (2002). The Psychological Impact of Incarceration: Implications for Post-Prison Adjustment. Washington, DC: U.S. Department of Health Human Services. Recuperado de https://aspe.hhs.gov/system/files/pdf/75001/Haney. pdf

Jiang, S. y Winfree, L. T. (2006). Social Support, Gender, and Inmate Adjustment to Prison Life Insights from a National Sample. The Prison Journal, $\quad 86, \quad 32-55$. https://doi.org/10.1177/0032885505283876

Jordan, M. (2011). The Prison Setting as a Place of Enforced Residence, it's Mental Health Effects, and the Mental Healthcare Implications. Health Place, 17, 1061-1066. https://doi.org/10.1016/j.healthplace.2011.06.006

Kirchner, T., Forns, M. y Mohíno, S. (2007). Psychological Adjustment in a Forensic Sample: Relationship with Approach- and AvoidanceCoping Typologies. Journal of Forensic Sciences, 52, 712-716. https://doi.org/10.1111/j.15564029.2007.00430.x iebling, A. y Crewe, B. (2012). Prison Life, Penal Power, and Prison Effects. En R. Morgan, M. Maguire y R. Reiner (Eds.), The Oxford Handbook of Criminology (pp. 895-927). Oxford, UK: OUP.

Moller, L., Stover, H., Jurgens, R., Gatherer, A. y Nikogosian, H. (2007). Health in Prison, a WHO Guide to the Essentials in Prison Health. Copenhagen, Dinamarca: World Health Organization Regional Office for Europe.

Moos, R. H. (1993). Coping Responses Inventory: Adult Form. Professional Manual. Odessa, FL: Psychological Assessment Resources.

Pastwa-Wojciechowska, B., Kaźmierczak, M. y Błażek, M. (2012). Self-esteem and Styles of Coping with Stress versus Strategies of Planning in People with Psychopathic Personality Disorders. Medical Science Monitor, 18, 119-124. https://doi.org/10.12659/MSM.882467

Paterline, B. A. y Petersen, D. M. (1999). Structural and Social Psychological Determinants of Prisonization. Journal of Criminal Justice, 27, 427-441. https://doi.org/10.1016/S0047-2352(99)00014-8

Pérez, B., Herrero, J., Velasco, J. y Rodríguez-Díaz, F. J. (2015). A Contrastive Analysis of the Factorial Structure of the PCL-R: Which Model Fits best the Data? European Journal of Psychology Applied to Legal Context, 7, 23-30. https://doi.org/10.1016/j.ejpal.2014.10.001

Picken, J. (2012). The Coping Strategies, Adjustment and Well Being of Male Inmates in Prison. Internet Journal of Criminology, 1-29. Recuperado de https://studylib.net/doc/8657392/the-copingstrategies--adjustment-and-well-being-of-male

Powell, J., Harris, F., Condon, L. y Kemple, T. (2010). Nursing Care of Prisoners: Staff Views and Experiences. Journal of Advanced Nursing, 66, 1257-1265. https://doi.org/10.1111/j.13652648.2010.05296.x 
Rocheleau, A. M. (2013). Ways of Coping and Involvement in Prison Violence. International Journal of Offender Therapy and Comparative Criminology, $\quad 59, \quad 359-383$ https://doi.org/10.1177/0306624X13510275

Sappington, A. A. (1996). Relationships among Prison Adjustment, Beliefs and Cognitive Coping Style. International Journal of Offender Therapy and Comparative Criminology, 40, 54-62. https://doi.org/10.1177/0306624X96401007

Smyth, N. J., Ivanoff, A. y Jang, S. J. (1994). Changes in Psychological Maladaptation among Inmate Parasuicides. Criminal Justice and Behavior, 21, 357-365.

https://doi.org/10.1177/0093854894021003005

Vitaliano, P. P., Russo, J. y Maiuro, R. D. (1987). Locus of Control, Type of Stressor, and Appraisal within a Cognitive-Phenomenological Model of Stress. Journal of Research in Personality, 21, 224-237. https://doi.org/10.1016/0092-6566(87)90009-2

Walker, J., Illingworth, C., Canning, A., Garner, E., Woolley, J. y Taylor, P. (2014). Changes in Mental State Associated with Prison Environments: A Systematic Review. Acta Psychiatrica Scandinavica, $\quad 129, \quad$ 427-436. https://doi.org/10.1111/acps.12221

Weiten, W., Dunn, D. y Hammer, E. (2011). Psychology Applied to Modern Life: Adjustment in the 21st Century. Belmont, CA: Thomson Learning.

Wolfgang, M. E. (1961). Quantitative Analysis of Adjustment to the Prison Community. The Journal of Criminal Law, Criminology, and Police Science,
51, 607-618. Recuperado de http://scholarlycommons.law.northwestern.edu/cgi/ viewcontent.cgi?article $=4991 \&$ context $=$ jcl

Yang, S., Kadouri, A., Révah-Lévy, A., Mulvey, E. P. y Falissard, B. (2009). Doing Time: A Qualitative study of long-term Incarceration and the Impact of Mental Illness. International Journal of Law and Psychiatry, 32, 294-303. https://doi.org/10.1016/j.ijlp.2009.06.003

Zabala-Baños, M. C., Segura, A., Maestre-Miquel, C., Martínez-Lorca, M., Rodríguez-Martín, B., Romero, D. y Rodríguez, M. (2016). Mental Disorder Prevalence and Associated Risk Factors in three Prisons of Spain. Revista Española de Sanidad Penitenciaria, 18 , https://dx.doi.org/10.4321/S157506202016000100003

Zamble, E. (1992). Behavior and Adaptation in longterm Prison Inmates: Descriptive Longitudinal Results. Criminal Justice and Behavior, 19, 409425. https://doi.org/10.1177/0093854892019004005

Zamble, E. y Porporino, F. J. (1988). Coping, Behavior, and Adaptation in Prison Inmates. New York, NY: Springer-Verlag.

Zamble, E. y Porporino, F. (1990). Coping, Imprisonment, and Rehabilitation: Some Data and their Implications. Criminal Justice and Behavior, 17 , 53-70. https://doi.org/10.1177/0093854890017001005 


\title{
ADAPTATION TO PRISON AND PSYCHOLOGICAL ADJUSTMENT IN A SAMPLE OF PRISON INMATES
}

\author{
MerCedes Novo ${ }^{1}$, ANA PEREIRA ${ }^{2}$, MARÍA JOSÉ VÁZQUEZ ${ }^{3}$ Y \\ BÁRBARA G. AMADO ${ }^{2}$
}

\section{EXTENDED SUMMARY}

\section{Introduction}

Though research has focused on prison institutionalization, the impact of prison environments on the mental health of prison inmates remains unclear. This underscores the need to analyse the adaptation to the prison context and its relationship with psychological adjustment and cognitive skills.

\section{Method}

\section{Participants}

A total of 112 inmates serving sentences in the Central de Paços de Ferreira Penitentiary (Portugal) participated in the study. All subjects were men, aged from 23 to 74 years $(M=40.40, D T=1.04), 77$ with previous criminal records and mostly Caucasian $(94 \%)$. As for marital status, $28.6 \%$ (32) lived as couples (married, common-law union, or consensual unions), and $71.4 \%$ (80) were (bachelors, separated, widowed).

The mean number of crimes committed in this sample was $8.57(D T=0.90)$, ranging from 1 to 44 crimes. As for the types of crime, the Portuguese criminal code describes crimes against society and property (34.85\%), people $(16 \%)$, society $(11.6 \%)$, society and people $(9.8 \%)$, property $(8 \%)$, property and people $(6.3 \%)$, with $13.4 \%$ of inmates serving sentences for two or more crimes. The sample was consisted of $61.60 \%$ reoffenders versus $38.46 \%$ first-time offenders. In short, most inmates had a criminal record and were serving sentences for crimes against people, society, and property.

\section{Procedure and design}

All subjects voluntarily participated in the study. The study design was approved by the penitentiaries and informed written consent was obtained from the inmates'. The design sensitivity analysis showed the probability of detecting $(1-\beta)$ significant differences $(\alpha<.05)$ for a mean effect size ranged from $84 \%$ to $99 \%$ according to data analysis. Consequently, the study design was highly sensitive to detecting significant differences.

\section{Measurement Instruments}

The inmates' sociodemographic and legal-penal data was gleaned from their prison records. As for the psychopathological measurement instrument, inmates were administered the SCL-90-R (Derogatis, 1994), that evaluates nine clinical dimensions and three global severity indicators. This instrument was back translated from Spanish to Portuguese.

For the participants in this study, the analysis of internal consistency of the SCL-90 showed high internal consistency for the clinical scales (i.e., for participants in the study, $\alpha=.801$ for somatization scale; $\alpha=.680$ for obsessive-compulsive; $\alpha=.731$ for interpersonal sensitivity; $\alpha=.716$ for depression; $\alpha=.771$ for anxiety; $\alpha$ 
$=.674$ for hostility; $\alpha=.579$ for phobic anxiety; $\alpha=.524$ for paranoid ideation, and $\alpha=.677$ for psychoticism).

To evaluate coping ability, the CRI-A (Coping Responses Inventory Adult Form) de Moos (1993) that consists of two parts, was completed. In both parts, inmates were instructed to respond to the test with their entry into prison as the situation-problem. The CRI-A evaluates eight types coping strategies that were shown to be reliable with the participants in this study: Logical analysis $(L A)(\alpha=.771)$; Positive Reappraisal $(P R)(\alpha=.638)$; Seeking Guidance and Support $(S G)(\alpha=.676)$; Problem Solving (PS) $(\alpha=.710) ;$ Cognitive Avoidance $(C A)$ $(\alpha=.790)$; Acceptance or Resignation $(A R)(\alpha=.602)$; Seeking Alternative Rewards $(S R)(\alpha=.793)$, and Emotional Discharge $(E D)(\alpha=.810)$.

To examine perceived social status, the MacArthur Scale of Subjective Social Status (Adler and Stewart, 2007) was applied where inmates use a 10-point scale to rank the position of the inmate in relation to other prison inmates to indicate perceived social status. The inmates' external social status was evaluated by requesting prison personnel to score each inmate on a 10-point scale of an adapted version based on social status criteria defined by Caldwell (1956). To assess the adaptation to prison, the Prison Adjustment Index (PAI) of Wolfgang (1961) was used to evaluate the degree of each inmate's adaptation from a perspective and with administrative criteria, provided by the prison personnel.

\section{Results}

\section{Adaptation to prison and criminologi- cal variables}

Inmates with a history of early behavioural problems showed significant differences in the level of adaptation, $t(110)=2.18, p<.05, d=0.48$, with less adaptation for these inmates $(M=0.43)$, as compared to inmates with no such history $(M=0.66)$. Moreover, inmates with problems of illegal substance abuse showed statistically significant differences in prison adaptation, $t(107.05)=3.07, p<.01, d=0.62$. Thus, inmates with a history of abuse is indicative of worse adaptation to prison $(M=0.45)$, as compared to inmates with no history of abuse $(M=0.73)$.

Furthermore, differences were observed in the adaptation of prison inmates according to family history of psycho-emotional problems, $t(90.19)=1.92, p<.05$, $d=0.40$. The inmates worst adapted to the prison context had a history of these family problems $(M=0.48)$, as compared to inmates without psycho-emotional problems $(M=0.67)$. Moreover, inmates with no partner $(M=0.50)$ adapted worse to prison than inmates with partners $(M=0.84), \mathrm{t}(77.47)=3.99, p<.001, d=0.77$. Thus, a history of risk behaviour, and early behavioural and psycho-emotional problems in the context of the family are associated to worse adjustment to the prison system, whereas having a relationship with a partner or married couples appears to be a protective factor against nonadaptation to prison.

The results also revealed a significant relationship between prison adaptation and external social status, $\chi^{2}(1$, $N=112)=3.57, p<.05, \mathrm{phi}=.18$. In other words, $68 \%$ of well adapted inmates had high external status as assigned by the prison personnel, in comparison to $32 \%$ of non-adapted inmates. Whereas $51 \%$ of low status inmates were classified as adapted, $49 \%$ were not adapted. Nevertheless, the discrepancy between the external evaluation of prison personnel and the inmates self-reported social status $t(112)=4.39, p<.001, d=0.42$, indicated inmates hold a positively biased self-perception of social status in prison $(M=1.01$, IC95\%[0.56, 1.46]), though variability was very high $(D T=2.43, \mathrm{VC}=240.59)$. This result would suggest that self-perceived social status is based on the compliance of the prison code and values, whereas external social status is based on the adaptation and adjustment to prison.

\section{Psychological adjustment}

In the population of prison inmates and the normalized population highlights that inmates, in comparison to the non-pathological population (contrast sample of delinquents, not mental patients), exhibited 
significant symptomology characteristic of somatization, obsessive-compulsive, interpersonal sensitivity, depressive, anxiety, hostility, phobic anxiety, paranoid ideation, and psychosis. In short, the prison population differed from the general population in all of the clinical variables of the SCL, and the three severity indices i.e., the former displayed more clinical symptomology and of greater intensity.

Regarding the condition of reoffending, it was not associated to clinical symptomology, save on the obsessive-compulsive scale, which was higher in prison inmates. Reoffenders suffered from more thoughts, impulses, and actions that are experienced as impossible to avoid and undesired. Moreover, the degree of prison adaptation had no influence on the clinical symptomology of inmates i.e., neither symptomology nor symptom severity were greater in relation to the degree of adaptation to prison.

\section{Cognitive skills for problems-solving}

Coping involves four problem approximation strategies (Logical analysis, Positive revaluation, Search for guidance and support, Problem-solving), and four de avoidance strategies (Cognitive Avoidance, Acceptance or Resignation, Seeking Alternative Rewards, Emotional Discharge). Significant differences were observed in all of the coping avoidance scales, indicating inmates used more avoidance strategies. Approximation strategies refer to differences exclusively in logical analysis, a strategy that inmates used less than the general population.

\section{Discussion}

This study is subject to several limitations which should be borne in mind in interpreting the results. First, the sample was homogeneous, and the effect of the maturation of the inmates through time was not controlled, and the impact of prison treatment on inmates benefiting from prison intervention programs. Moreover, this type of population tends to manipulate responses both towards feigning to avoid sanctions or to obtain their prison clas- sification, and defensiveness, either aimed at concealing negative characteristics or to exhibit positive characteristics to obtain prison benefits. Taking into account these limitations that mediate the generalization of the results, the following conclusions may be drawn.

As for status and the different approaches to evaluating it, que, from the personnel' perspective, inmates obtaining high social status were better adapted. Notwithstanding, a discrepancy was observed between selfreported social status and external evaluation, whereby inmates have a positively biased perception of their status social. In relation to the prison adaptation of inmates, the prison context was found to lead to greater maladjustment in vulnerable subjects, for instance inmates who are illegal substance abusers, and those with psychoemotional problems in the family context or early behavioural problems, whereas having a partner improved adaptation. Thus, the results substantiate the importing model that contends the prison culture imports habits, values, and customs that have been previously acquired by inmates prior to entering prison and influence the level of prisonization.

Regarding psychological adjustment, the results indicate the prison population is different to the general population in all of the clinical variables under evaluation, and the three severity indices i.e., inmates display more clinical symptomology and of a greater severity, even though it may not be related to the degree of prison adaptation. This result corroborated the findings of previous studies that found a greater prevalence of psychopathological disorders in the prison population and the greater risk of depression. These changes and adaptations seem to respond to the process of prisonization. Thus, several authors have reported deterioration in the mental health of inmates when they enter prison, but improvement in mental health is observed through the period of incarceration, particularly after the first year and in small prisons. The prison system should be responsible for caring for the mental health of inmates and should seek to take advantage of a prisoner's admittance to prison as an opportunity for intervention and for improving the health of inmates in accordance with the principles of Therapeutic Jurisprudence. Moreover, further research is 
required to examine the relationship between mental health and the prison system as a key objective.

Furthermore, the results of this study have revealed that inmates used more avoidance strategies i.e., cognitive avoidance, acceptance or resignation, search for alternative rewards, and emotional unloading. This avoidance coping style centred on emotions is characterized by the tendency to illusions, thought avoidance and to conceiving these situations as unreal, and to foster negative emotions such as rage. As for approximation strategies, inmates resort less to logical analysis than the general population. Hence, the personal risk that is characteristic of the prison context inhibits any sign of vulnerability and fosters the use of approximation coping strategy leading to maladjustment inmates.

In short, this study has contributed to the assessment of the adaptation and adjustment of prison inmates and is relevant to the design of prison intervention programs and prisoner release programs that have proven to be efficacious in improving social and cognitive competence, and in reducing the effects of prisonization (Dettbarn, 2012). 